Category: Other diseases (including muscle) and Diagnostics

\title{
Reducing unplanned hospital admissions in patients with neuromuscular diseases: an NHS Re-audit
}

Renata S. Scalco, Laura Nastasi, Michael G. Hanna, Ros Quinlivan

MRC Centre for Neuromuscular Diseases, UCL, London, UK

r.scalco@ucl.ac.uk

Background: Patients with neuromuscular diseases (NMDs) require long-term multidisciplinary care. Access to specialised NMD services is still variable across the UK. In 2012, an audit showed a high frequency of emergency admissions in this patient population, which were more likely to happen if care was poorly co-ordinated and not proactive in preventing NMDs complications. The audit was followed by several recommendations and a partnership approach project.

Aims: To assess the impact of the previous Audit

Methods: A re-audit was undertaken across the same specialised commissioning groups in Central and South-East England.

Results: More patients were known to specialised centres in NMDs, and the majority of admissions were under the care of Neurosciences (77\%) as compared to 2012 when only $14.9 \%$ were admitted under Neuroscience. Improvements included a reduction in preventable admissions directly related to known NMDs (from $63 \%$ to $32.8 \%$ ) and reduction in re-admissions (from $25.1 \%$ to $12.4 \%$ ). The mortality rate dropped from $4.5 \%$ to $0.3 \%$. Patients known to a NMD specialised service had shorter hospital stay and fewer ITU admissions than patients who were not known to specialised services.

Conclusion: The re-audit on unplanned hospital admissions in England highlighted the importance of co-ordination of care at specialised services for patients with neuromuscular disorders. It successfully documented improvements in the care provided to patients with NMDs in the assessed regions. 